\title{
On a Poisson summation formula for noncommutative tori
}

\author{
Igor Nikolaev
}

\begin{abstract}
It is proved that a maximal abelian subalgebra of the noncommutative torus commutes with the Laplace operator on a complex torus. As a corollary, one gets an analog of the Poisson summation formula for noncommutative tori.
\end{abstract}

Key words and phrases: Selberg trace formula, noncommutative torus

MSC: 11F72 (Selberg trace formula); $46 L 85$ (noncommutative topology)

\section{Introduction}

The Poisson summation formula is an elementary and fundamental fact of harmonic analysis and representation theory. The simplest case of such a formula says that for every function $f \in C_{0}^{\infty}(\mathbb{R})$ it holds

$$
\sum_{n \in \mathbb{Z}} f(n)=\sum_{n \in \mathbb{Z}} \hat{f}(n),
$$

where $C_{0}^{\infty}(\mathbb{R})$ is the set of $C^{\infty}$-smooth complex-valued functions with a compact support on the real line $\mathbb{R}$ and $\hat{f}(\nu):=\int_{-\infty}^{\infty} f(x) e^{-2 \pi i \nu x} d x$ is the Fourier transform of $f$. Let $\mathbb{H}=\{x+i y \in \mathbb{C} \mid y>0\}$ be the Lobachevsky halfplane and $\tau \in \mathbb{H}$; an analog of formula (11) for the two-dimensional lattice $L_{\tau}=\mathbb{Z}+\tau \mathbb{Z}$ can be written as

$$
\sum_{m \in \mathbb{Z}} \sum_{n \in \mathbb{Z}} f\left(m^{2}+2 m n \Re(\tau)+n^{2}|\tau|^{2}\right)=\sum_{r=0}^{\infty} \lambda(r) \hat{f}(r),
$$


where function $f \in C_{0}^{\infty}\left(\mathbb{R}^{2}\right)$ is radially symmetric (i.e. $f(u, v)=$ Const on $\left.u^{2}+v^{2}=r \geq 0\right)$ while $\lambda(r):=\left|\left\{(m, n) \in \mathbb{Z}^{2}: m^{2}+n^{2}=r\right\}\right|$ the multiplicity function and $\hat{f}$ the Fourier transform of $f$ given by the formula

$$
\hat{f}(r)=\frac{\pi}{\Im(\tau)} \int_{0}^{\infty} f(s) J_{0}\left[2 \pi \sqrt{s} \sqrt{\frac{m^{2}|\tau|^{2}-2 m n \Re(\tau)+n^{2}}{\Im^{2}(\tau)}}\right] d s,
$$

with $J_{0}(z)=\frac{1}{\pi} \int_{0}^{\pi} \cos (z \cos \alpha) d \alpha$ being the Bessel function.

Recall that each radially symmetric function $f \in C_{0}^{\infty}\left(\mathbb{R}^{2}\right)$ gives rise to a symmetric Hilbert-Schmidt integral operator on the Hilbert space $L^{2}\left(\mathbb{C} / L_{\tau}\right)$ acting by the formula:

$$
\left(T_{f} \varphi\right)(z)=\int_{\mathbb{C} / L_{\tau}} f(z, w) \varphi(w) d w
$$

where $f(z, w)=\sum_{z_{0} \in L_{\tau}} f\left(z+z_{0}, w\right)$, see e.g. [Iwaniec 1995] [4], p. 5. Denote by $\sum f\left(L_{\tau}\right)$ the LHS of (2); a link between $T_{f}$ and the Poisson summation is given by the formula

$$
\operatorname{tr}\left(T_{f}\right)=\sum f\left(L_{\tau}\right),
$$

where $\operatorname{tr}$ is the trace of $T_{f}$. The $T_{f}$ commutes with the Laplace operator $\Delta=\frac{\partial^{2}}{\partial x^{2}}+\frac{\partial^{2}}{\partial y^{2}}$ on the complex torus $\mathbb{C} / L_{\tau}$ and operators $T_{f}$ commute with each other for all $f \in C_{0}^{\infty}(\mathbb{R})$, ibid. It is easy to see, that complex conjugation defines an adjoint operator $T_{f}^{*}=T_{\bar{f}}$. The norm closure of the $*$-algebra generated by all $T_{f}$ is a commutative $C^{*}$-algebra, see e.g. [Murphy 1990] [5] for an introduction; such a $C^{*}$-algebra we denote by

$$
\mathcal{R}\left(\mathbb{C} / L_{\tau}\right):=\overline{\left\{T_{f}: f \in C_{0}^{\infty}\left(\mathbb{R}^{2}\right)\right\}} .
$$

In this note we construct an inclusion of the algebra $\mathcal{R}\left(\mathbb{C} / L_{\tau}\right)$ into a noncommutative torus $\mathcal{A}_{\theta}$, i.e. the $C^{*}$-algebra generated by unitary operators $u$ and $v$ satisfying the commutation relation $v u=e^{2 \pi i \theta} u v$ for a constant $\theta \in \mathbb{R}$ [Rieffel 1990] [10]. Namely, let $\left[a_{0}, a_{1}, \ldots\right]$ be the regular continued fraction of $\theta$ and consider the Bratteli diagram in Figure 1, where $a_{i}$ is the multiplicity of edges of the diagram [Bratteli 1972] [1]. (Note that the diagram is infinite, unless $\theta$ is a rational number; in this case the canonical trace on $\mathcal{A}_{\theta}$ is defined by continuity from the irrational values of $\theta$.) Let $X_{\theta}$ be the Bratteli compactum, i.e. a Cantor set obtained from the infinite paths of the Bratteli diagram, see [Herman, Putnam \& Skau 1992] [3], pp. 837-838. We shall denote by $\frac{1}{\mu} X_{\theta}$ the Cantor set $X_{\theta}$ endowed with the measure $\mu$ and by $C\left(\frac{1}{\mu} X_{\theta}\right)$ 


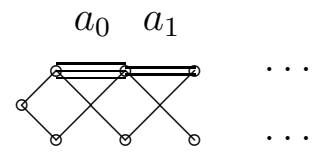

Figure 1: Bratteli diagram of $\mathcal{A}_{\theta}$.

the commutative $C^{*}$-algebra of complex-valued functions on $\frac{1}{\mu} X_{\theta}$. We shall write $\left(\mathcal{A}_{\theta}, \frac{1}{\mu} e\right)=F\left(\mathbb{C} / L_{\tau}\right)$ to denote the noncommutative torus $\mathcal{A}_{\theta}$ with a scaled unit $\frac{1}{\mu} e$ corresponding to the complex torus $\mathbb{C} / L_{\tau}$ under a functor $F$, see Section 2.1. Our main results can be stated as follows.

Theorem 1 There exists a trace-preserving isomorphism:

$$
\mathcal{R}\left(\mathbb{C} / L_{\tau}\right) \cong C\left(\frac{1}{\mu} X_{\theta}\right) \subset\left(\mathcal{A}_{\theta}, \frac{1}{\mu} e\right),
$$

where $C\left(\frac{1}{\mu} X_{\theta}\right)$ is the maximal abelian subalgebra of the $C^{*}$-algebra $\left(\mathcal{A}_{\theta}, \frac{1}{\mu} e\right)$.

Corollary 1 (Poisson summation formula) For each radially symmetric function $f \in C_{0}^{\infty}\left(\mathbb{R}^{2}\right)$ there exists a Hilbert-Schmidt operator $T_{f} \in\left(\mathcal{A}_{\theta}, \frac{1}{\mu} e\right)$, such that:

$$
\sum f\left(L_{\tau}\right)=\operatorname{tr}\left(T_{f}\right)
$$

where $\operatorname{tr}$ is the canonical trace on $\left(\mathcal{A}_{\theta}, \frac{1}{\mu} e\right)$ [Rieffel 1981] [9]. The operator $T_{f}$ is self-adjoint if and only if $f$ is a real-valued function.

Remark 1 Theorem 1 says that the $C^{*}$-algebra $\mathcal{R}\left(\mathbb{C} / L_{\tau}\right)$ is a maximal abelian subalgebra (masa) of the noncommutative torus $\left(\mathcal{A}_{\theta}, \frac{1}{\mu} e\right)=F\left(\mathbb{C} / L_{\tau}\right)$; the masa determines the $C^{*}$-algebra $\left(\mathcal{A}_{\theta}, \frac{1}{\mu} e\right)$ itself by taking the crossed product of $C\left(\frac{1}{\mu} X_{\theta}\right)$ with the Vershik homeomorphism of the Cantor set $\frac{1}{\mu} X_{\theta}$, see Section 2.2.

The structure of the article is as follows. We recall some useful facts in Section 2. Theorem 1 and corollary 1 are proved in Section 3. 


\section{Preliminaries}

We briefly review a relation between complex and noncommutative tori, the $C^{*}$-dynamical systems on the Cantor set and the Selberg trace formula. For an extended account and details we refer the reader to [6], [Herman, Putnam \& Skau 1992] [3] and [Iwaniec 1995] [4], respectively.

\subsection{Complex and noncommutative tori}

Recall that $\mathbb{C} / L_{\tau}$ is isomorphic to the intersection two quadric surfaces in the complex projective space of the form $\left\{(u, v, w, z) \in \mathbb{C} P^{3} \mid u^{2}+v^{2}+w^{2}+z^{2}=\right.$ $\left.\frac{1-\alpha}{1+\beta} v^{2}+\frac{1+\alpha}{1-\gamma} w^{2}+z^{2}=0\right\}$, where $\alpha, \beta, \gamma$ are some complex constants such that $\alpha+\beta+\gamma+\alpha \beta \gamma=0$. It was proved by Sklyanin that a free $\mathbb{C}$-algebra $S_{\alpha, \beta, \gamma}$ on four generators $x_{i}$ and six quadratic relations

$$
\left\{\begin{array}{c}
x_{1} x_{2}-x_{2} x_{1}=\alpha\left(x_{3} x_{4}+x_{4} x_{3}\right), \\
x_{1} x_{2}+x_{2} x_{1}=x_{3} x_{4}-x_{4} x_{3}, \\
x_{1} x_{3}-x_{3} x_{1}=\beta\left(x_{4} x_{2}+x_{2} x_{4}\right), \\
x_{1} x_{3}+x_{3} x_{1}=x_{4} x_{2}-x_{2} x_{4}, \\
x_{1} x_{4}-x_{4} x_{1}=\gamma\left(x_{2} x_{3}+x_{3} x_{2}\right), \\
x_{1} x_{4}+x_{4} x_{1}=x_{2} x_{3}-x_{3} x_{2},
\end{array}\right.
$$

is a coordinate ring of complex torus $\mathbb{C} / L_{\tau}$, i.e. $\operatorname{Mod}\left(S_{\alpha, \beta, \gamma}\right) /$ Tors $\cong$ Coh $\left(\mathbb{C} / L_{\tau}\right)$, where Coh is the category of quasi-coherent sheaves on $\mathbb{C} / L_{\tau}$, Mod the category of graded left modules over the graded ring $S_{\alpha, \beta, \gamma}$ and Tors the full sub-category of Mod consisting of the torsion modules, see e.g. [Smith \& Stafford 1993] [11], p.267. The closure of a self-adjoint representation of the Sklyanin algebra $S_{\alpha, \beta, \gamma}$ by linear operators on a Hilbert space $\mathcal{H}$ is isomorphic to the algebra $\mathcal{A}_{\theta}$ with a scaled unit $\frac{1}{\mu}$ e for a constant $\mu>0$. The bijection $F: \mathbb{C} / L_{\tau} \mapsto\left(\mathcal{A}_{\theta}, \frac{1}{\mu} e\right)$ is a functor from isomorphic complex tori to the stably isomorphic noncommutative tori [6, Section 1.3].

\section{$2.2 C^{*}$-dynamical systems on the Cantor set}

An $A F$-algebra (approximately finite-dimensional $C^{*}$-algebra) is defined to be the norm closure of an ascending sequence of the finite-dimensional $C^{*}$ algebras $M_{n}$ 's, where $M_{n}$ is the $C^{*}$-algebra of the $n \times n$ matrices with the entries in $\mathbb{C}$. To describe the ascending sequence, we use an infinite graph 
called a Bratteli diagram of the $A F$-algebra [Bratteli 1972] [1]. The Bratteli diagram defines a unique $A F$-algebra. By an ordered Bratteli diagram one understands a natural (partial) order between the edges of the diagram defined as follows. Two edges $e$ and $e^{\prime}$ are comparable, if and only if, there exist two paths through $e$ and $e^{\prime}$ respectively, which have an edge in common; in this case one writes $e>e^{\prime}$ whenever $e$ lies above $e^{\prime}$ on the diagram; we refer the reader to [Herman, Putnam \& Skau 1992] [3], pp. 835-836 for the details.

To each Bratteli diagram one assigns a Cantor set, i.e. the compact totally disconnected metric space $X$ consisting of the infinite paths of the diagram. Let $P_{0, k}$ denote a path $\left(e_{0}, \ldots, e_{k}\right)$ consisting of $k+1$ edges $e_{i}$ of the Bratteli diagram. The inverse limit $X=\lim P_{0, k}$ is a Cantor set defined by the discrete topology on $P_{0, k}$, see [Herman, Putnam \& Skau 1992] [3], pp. 837-838. The Cantor set $X$ is called a Bratteli compactum.

The ordered Bratteli diagram gives rise to a homeomorphism $\varphi: X \rightarrow X$ of the Bratteli compactum $X$ associated to the diagram. Roughly speaking, $\varphi$ sends an infinite path of the Bratteli diagram (i.e. a point of $X$ ) to the same infinite path but with a finite number of edges replaced by the successor edges defined by the ordering, see [Herman, Putnam \& Skau 1992] [3], p. 838. The map $\varphi$ is called a Vershik homomorphism of the Bratteli compactum $X$. The iterations of $\varphi$ define a minimal dynamical system $(X, \varphi)$ on the Cantor set $X$. By a $C^{*}$-dynamical system on the Cantor set $X$ one understands the crossed product $C^{*}$-algebra $C(X) \rtimes_{\varphi} \mathbb{Z}$, where $C(X)$ is the $C^{*}$-algebra of complex-valued functions on $X$. (This is true also for the rational values of $\theta$.)

Lemma $1 C(X) \rtimes_{\varphi} \mathbb{Z} \subset \mathbb{A}$, where $\mathbb{A}$ is the AF-algebra defined by the Bratteli diagram upon which the Cantor set $X$ was constructed.

Proof. See [Putnam 1989] [8], pp. 346-350.

\subsection{Selberg trace formula}

To put the Poisson summation formula in a general context, consider the homogeneous space $G / K$, where $G \cong S L(2, \mathbb{R})\left(G \cong \mathbb{R}^{2}\right.$, resp. $)$ and $K \cong$ $S O(2, \mathbb{R})$ ( $K$ is trivial, resp.) are the Lie groups; thus $G / K \cong \mathbb{H}$ is the Lobachevsky half-plane $\left(G / K \cong \mathbb{R}^{2}\right.$ is the Euclidean plane, resp.) Recall that the left regular representation of $G$ gives rise to a linear operator $\left(T_{\gamma} f\right)(z)=f(\gamma z)$ on the space of complex-valued functions $f: G \rightarrow \mathbb{C}$. 
A linear operator $T$ is said to be invariant if it commutes with $T_{\gamma}$, i.e. $T(f(\gamma z))=(T f)(\gamma z), \quad \forall \gamma \in G$.

Example 1 The Laplace-Beltrami operator $\Delta=y^{2}\left(\frac{\partial^{2}}{\partial x^{2}}+\frac{\partial^{2}}{\partial y^{2}}\right)$ (the Laplace operator $\Delta=\frac{\partial^{2}}{\partial x^{2}}+\frac{\partial^{2}}{\partial y^{2}}$, resp.) is an invariant differential operator on $C^{\infty}(G / K)$.

Example 2 Consider the integral operator $\left(T_{f} \varphi\right)(z)=\int_{G / K} f(z, w) \varphi(w) d w$, where the kernel $f: G / K \times G / K \rightarrow \mathbb{C}$ satisfies condition $f(\gamma z, \gamma w)=f(z, w)$ for all $\gamma \in G$; the $T_{f}$ is an invariant integral operator and such operators commute with each other.

Lemma 2 The invariant integral operators $T_{f}$ commute with the LaplaceBeltrami operator $\Delta$ (the Laplace operator $\Delta$, resp.); moreover, any eigenfunction of $\Delta$ coincides with an eigenfunction of all invariant integral operators.

Proof. See e.g. [Iwaniec 1995] [4], pp. 29-33.

Let $\Gamma$ be a discrete subgroup of $S L(2, \mathbb{R})$ (a lattice $L_{\tau}=\mathbb{Z}+\mathbb{Z} \tau$ of $\mathbb{R}^{2} \cong \mathbb{C}$, resp.); then $\Gamma \backslash \mathbb{H}$ is a Riemann surface $\left(\mathbb{C} / L_{\tau}\right.$ is a complex torus, resp.) A function $f: G / K \rightarrow \mathbb{C}$ is said to be automorphic with respect to $\Gamma$ if it satisfies the periodicity condition $f(\gamma z)=f(z)$ for all $\gamma \in \Gamma$; we shall denote the space of such functions by $A(G / K)$. Each function $f: G / K \rightarrow \mathbb{C}$ with a rapid decay on $G / K$ (e.g. a radially symmetric function $f \in C_{0}^{\infty}(G / K)$ ) gives rise to an automorphic function with respect to $\Gamma$ given by the formula $f(z):=\sum_{\gamma \in \Gamma} f(\gamma z)$.

Further we restrict the Laplace operator $\Delta$ to the space $A(G / K)$ and consider the invariant integral operators $T_{f}$ on $A(G / K)$ given by the formula

$$
\left(T_{f} \varphi\right)(z)=\int_{\Gamma \backslash G / K} f(z, w) \varphi(w) d w,
$$

where $f(z, w)=\sum_{\gamma \in \Gamma} f\left(z^{-1} \gamma w\right)$; it is known that $T_{f}$ are compact trace class operators and lemma 2 is valid for them. Moreover, the operators $T_{f}$ satisfy the following remarkable formula.

Lemma 3 (Selberg trace formula) If $T_{f}$ is a self-adjoint operator, then

$$
\operatorname{tr}\left(T_{f}\right)=\sum_{[\gamma] \in \Gamma} \int_{F_{\gamma}} f([\gamma]) d z
$$


where $[\gamma]:=\left\{\tau^{-1} \gamma \tau \mid \tau \in \Gamma\right\}$ is the conjugacy class of $\gamma$ and $F_{\gamma}$ is the fundamental domain of the centralizer of $\gamma$.

Proof. See e.g. [Iwaniec 1995] [4].

Remark 2 The Selberg trace formula for $G \cong \mathbb{C}$ and $\Gamma=L_{\tau}$ coincides with the Poisson summation formula. Indeed, the LHS of (11) is equal to the $\sum \hat{f}\left(L_{\tau}\right)$, where $\hat{f}$ is the Fourier transform of $f$. The RHS of (11) corresponds to the $\operatorname{sum} \sum f\left(L_{\tau}\right)$.

\section{Proofs}

\subsection{Proof of theorem 1}

We shall split the proof in a series of lemmas.

Lemma $4 \mathcal{R}\left(\mathbb{C} / L_{\tau}\right)$ is commutative $C^{*}$-subalgebra of an $A F$-algebra $\mathbb{A}$.

Proof. Let $\mathcal{R}_{0}\left(\mathbb{C} / L_{\tau}\right)$ be a dense $*$-subalgebra of the $C^{*}$-algebra $\mathcal{R}\left(\mathbb{C} / L_{\tau}\right)$ consisting of the Hilbert-Schmidt integral operators $T_{f}$, where $f(r) \in C_{0}^{\infty}(\mathbb{R})$ is a radially symmetric function.

Denote by $V_{i} \subset L^{2}\left(\mathbb{C} / L_{\tau}\right)$ the eigenspace of a Hilbert-Schmidt operator $T_{f} \in \mathcal{R}_{0}\left(\mathbb{C} / L_{\tau}\right)$ corresponding to an eigenvalue $\lambda_{i}$. It is well known that $\operatorname{dim} V_{i}:=n_{i}<\infty$ and $V_{i}$ is a common eigenspace for all operators $T_{f} \in$ $\mathcal{R}_{0}\left(\mathbb{C} / L_{\tau}\right)$, see e.g. [Iwaniec 1995] [4], p.210. (The $V_{i}$ is also an eigenspace for the Laplace operator $\Delta$.)

A restriction of the algebra $\mathcal{R}_{0}\left(\mathbb{C} / L_{\tau}\right)$ to the subspace $V_{i}$ is a commutative subalgebra of the matrix algebra $M_{n_{i}}(\mathbb{C})$.

Denote by $\lambda_{1}>\lambda_{2}>\ldots$ the sequence of eigenvalues of the HilbertSchmidt operator $T_{f}$, see [Iwaniec 1995] [4], p.210; let $\left\{V_{1}, V_{2}, \ldots\right\}$ be the corresponding eigenspaces of dimensions $\left\{n_{1}, n_{2}, \ldots\right\}$, respectively. Consider the finite-dimensional $C^{*}$-algebra

$$
\mathbb{A}_{i}=M_{n_{1}}(\mathbb{C}) \oplus \ldots \oplus M_{n_{i}}(\mathbb{C}) .
$$

Similarly, the restriction of $\mathcal{R}_{0}\left(\mathbb{C} / L_{\tau}\right)$ to the sum of vector spaces $V_{1} \oplus \ldots \oplus$ $V_{i} \subset L^{2}\left(\mathbb{C} / L_{\tau}\right)$ is a commutative subalgebra of the $C^{*}$-algebra $\mathbb{A}_{i}$.

One gets an infinite ascending sequence of the finite-dimensional $C^{*}$ algebras $\mathbb{A}_{1} \subset \mathbb{A}_{2} \subset \ldots$; the corresponding $A F$-algebra we shall denote by A. 
Because $\cup \mathbb{A}_{i}$ is a dense subalgebra of $\mathbb{A}$, the closure of the $*$-algebra $\mathcal{R}_{0}\left(\mathbb{C} / L_{\tau}\right)$ defines a commutative $C^{*}$-subalgebra of the $A F$-algebra $\mathbb{A}$; in other words, the commutative $C^{*}$-algebra $\mathcal{R}\left(\mathbb{C} / L_{\tau}\right)$ is a subalgebra of the $A F$-algebra $\mathbb{A}$. Lemma 4 follows.

Lemma 5 The algebra $\mathcal{R}\left(\mathbb{C} / L_{\tau}\right) \cong C(X)$, where $X$ is the Bratteli compactum of algebra $\mathbb{A}$.

Proof. Because $\mathcal{R}\left(\mathbb{C} / L_{\tau}\right)$ is a commutative $C^{*}$-algebra, one gets by the Gelfand theorem that $\mathcal{R}\left(\mathbb{C} / L_{\tau}\right) \cong C\left(X_{0}\right)$, where $X_{0}$ is a compact Hausdorff topological space.

On the other hand, we showed earlier that the algebra $\mathcal{R}\left(\mathbb{C} / L_{\tau}\right)$ defines the $A F$-algebra $\mathbb{A}$, see lemma 4 .

The only commutative $C^{*}$-subalgebra of $\mathbb{A}$ with such a property is the $C^{*}$-algebra $C(X)$, where $X$ is the Bratteli compactum of $\mathbb{A}$, see [Herman, Putnam \& Skau 1992] [3], Theorem 8.8.

Therefore $X=X_{0}$ and lemma 5 follows.

Remark $3 \mathcal{R}\left(\mathbb{C} / L_{\tau}\right)$ is an $A F$-algebra.

Proof. In view of lemma 5, we have $\mathcal{R}\left(\mathbb{C} / L_{\tau}\right) \cong C(X)$, where $X$ is a Cantor set. It is known, that in this case $\mathcal{R}\left(\mathbb{C} / L_{\tau}\right)$ is an $A F$-algebra, see e.g. [Herman, Putnam \& Skau 1992] [3], p. 831. Remark 3 follows.

Lemma $6 \mathcal{R}\left(\mathbb{C} / L_{\tau}\right) \cong C\left(\frac{1}{\mu} X_{\theta}\right)$.

Proof. Consider a crossed product

$$
\mathcal{R}\left(\mathbb{C} / L_{\tau}\right) \rtimes \mathbb{Z} \subset \mathbb{A}
$$

by the Veshik homeomorphism of the Bratteli compactum $X$ of the $A F$ algebra $\mathbb{A}$; the crossed product is not an $A F$-algebra but its ordered $K_{0}$-group is isomorphic to such of $\mathbb{A}$, see [Herman, Putnam \& Skau 1992] [3].

On the other hand, it is known that the $A F$-algebra is defined up to an isomorphism by its ordered $K_{0}$-group, see [Elliott 1976] [2]. The crossed product (13) has a unique canonical trace; therefore one gets a dense abelian subgroup of the real line of the form:

$$
K_{0}\left(\mathcal{R}\left(\mathbb{C} / L_{\tau}\right) \rtimes \mathbb{Z}\right) \cong \mathbb{Z} \lambda_{1}+\ldots+\mathbb{Z} \lambda_{k}
$$


where $\lambda_{i} \in \mathbb{R}$ are some constants and the positive cone is defined by the inequality $\mathbb{Z} \lambda_{1}+\ldots+\mathbb{Z} \lambda_{k}>0$. But the algebra $\mathcal{R}\left(\mathbb{C} / L_{\tau}\right)$ depends on the complex modulus $\tau=x+i y \in \mathbb{H}$ whose real dimension is 2 ; therefore in formula (14) we have $k=2$, i.e. only $\lambda_{1}$ and $\lambda_{2}$ are independent parameters.

Put $\mu=\lambda_{1}>0$ and $\theta=\frac{\lambda_{2}}{\lambda_{1}}$. Then

$$
K_{0}\left(\mathcal{R}\left(\mathbb{C} / L_{\tau}\right) \rtimes \mathbb{Z}\right) \cong \mu(\mathbb{Z}+\mathbb{Z} \theta) \cong K_{0}\left(\left(\mathbb{A}_{\theta}, \frac{1}{\mu} e\right)\right),
$$

where $\mathbb{A}_{\theta}$ is the $A F$-algebra defined by the Bratteli diagram in Figure 1. But $K_{0}\left(\left(\mathbb{A}_{\theta}, \frac{1}{\mu} e\right)\right) \cong K_{0}\left(C\left(\frac{1}{\mu} X_{\theta}\right) \rtimes \mathbb{Z}\right)$ and comparing with (15) one gets a tracepreserving isomorphism $\mathcal{R}\left(\mathbb{C} / L_{\tau}\right) \cong C\left(\frac{1}{\mu} X_{\theta}\right)$ of the corresponding masas; this fact follows from the strong orbit equivalence of Cantor minimal systems established in [Herman, Putnam \& Skau 1992] [3] and Elliott's description of the Choquet simplex of the tracial states on the $A F$-algebras [Elliott 1976] [2]. Lemma 6 follows.

Lemma 7 The $C\left(\frac{1}{\mu} X_{\theta}\right)$ is a maximal abelian subalgebra of the $C^{*}$-algebra $\left(\mathcal{A}_{\theta}, \frac{1}{\mu} e\right)$.

Proof. Recall that

$$
C\left(\frac{1}{\mu} X_{\theta}\right) \rtimes_{\varphi} \mathbb{Z} \subset\left(\mathbb{A}_{\theta}, \frac{1}{\mu} e\right) \subset\left(\mathcal{A}_{\theta}, \frac{1}{\mu} e\right),
$$

where $\varphi$ is the Vershik homeomorphism, see [Putnam 1989] [8], Theorem 6.7 for the first inclusion and [Pimsner \& Voiculescu 1980] [7] for the second inclusion. On the other hand, the maximal abelian subalgebra of $C\left(\frac{1}{\mu} X_{\theta}\right) \rtimes_{\varphi}$ $\mathbb{Z}$ is isomorphic to the $C^{*}$-algebra $C\left(\frac{1}{\mu} X_{\theta}\right)$. Thus one gets the following inclusions

$$
C\left(\frac{1}{\mu} X_{\theta}\right) \subset C\left(\frac{1}{\mu} X_{\theta}\right) \rtimes_{\varphi} \mathbb{Z} \subset\left(\mathbb{A}_{\theta}, \frac{1}{\mu} e\right) \subset\left(\mathcal{A}_{\theta}, \frac{1}{\mu} e\right) .
$$

On the other hand, it is known that $K_{0}\left(X_{\theta} \rtimes_{\varphi} \mathbb{Z}\right) \cong K_{0}\left(\mathbb{A}_{\theta}\right) \cong K_{0}\left(\mathcal{A}_{\theta}\right)$. Therefore it follows from (17) that $C\left(\frac{1}{\mu} X_{\theta}\right)$ is a maximal abelian subalgebra of the larger algebra $\left(\mathcal{A}_{\theta}, \frac{1}{\mu} e\right)$. Lemma 7 follows.

Theorem 1 follows from lemmas 6 and 7 . 


\subsection{Proof of corollary 1}

Let $f \in C_{0}^{\infty}(\mathbb{R})$ be a radially symmetric function on the lattice $L_{\tau}$. Since the function is compactly supported, it defines a Hilbert-Schmidt integral operator $T_{f}$ on the Hilbert space $L^{2}\left(\mathbb{C} / L_{\tau}\right)$. In view of (5), one gets

$$
\sum f\left(L_{\tau}\right)=\operatorname{tr}\left(T_{f}\right)
$$

On the other hand, theorem 1 says that $T_{f} \in\left(\mathcal{A}_{\theta}, \frac{1}{\mu} e\right)$. It remains to show that $\operatorname{tr}\left(T_{f}\right)$ coincides with the canonical trace on the noncommutative torus.

Indeed, take a representation of $T_{f}$ on the Hilbert space $L^{2}\left(\mathbb{C} / L_{\tau}\right)$ such that the algebra $\mathcal{R}\left(\mathbb{C} / L_{\tau}\right)$ has a unique trace. Then the noncommutative torus $\left(\mathcal{A}_{\theta}, \frac{1}{\mu} e\right)$ admits a representation on the same space. These two representation are different in general, but always unitarily equivalent. In partic-

ular, the value of $\operatorname{tr}\left(T_{f}\right)$ is preserved for any unitary transformation of the space $L^{2}\left(\mathbb{C} / L_{\tau}\right)$. Corollary 1 follows.

\section{References}

[1] O. Bratteli, Inductive limits of finite dimensional $C^{*}$-algebras, Trans. Amer. Math. Soc. 171 (1972), 195-234.

[2] G. A. Elliott, On the classification of inductive limits of sequences of semisimple finite-dimensional algebras, J. Algebra 38 (1976), 29-44.

[3] R. H. Herman, I. F. Putnam and C. F. Skau, Ordered Bratteli diagrams, dimension groups and topological dynamics, International J. Math. 3 (1992), 827-864.

[4] H. Iwaniec, Introduction to the Spectral Theory of Automorphic Forms, Revista Mathemâtica Iberoamericana, 1995.

[5] G. J. Murphy, $C^{*}$-Algebras and Operator Theory, Academic Press, 1990.

[6] I. V. Nikolaev, Noncommutative Geometry, De Gruyter Studies in Math. 66, Berlin, 2017.

[7] M. Pimsner and D. Voiculescu, Imbedding the irrational rotation $C^{*}$ algebra into an AF-algebra, J. Operator Theory 4 (1980), 201-210. 
[8] I. F. Putnam, The $C^{*}$-algebras associated with minimal homeomorphisms of the Cantor set, Pacific J. Math. 136 (1989), 329-353.

[9] M. A. Rieffel, $C^{*}$-algebras associated with irrational rotations, Pacific J. of Math. 93 (1981), 415-429.

[10] M. A. Rieffel, Non-commutative tori - a case study of non-commutative differentiable manifolds, Contemp. Math. 105 (1990), 191-211.

[11] S. P. Smith and J. T. Stafford, Regularity of the four dimensional Sklyanin algebra, Compositio Math. 83 (1992), 259-289.

Department of Mathematics and Computer Science, St. John's University, 8000 Utopia Parkway, New York, NY 11439, United STATES; E-MAIL: igor.v.nikolaev@gmail.com 